\title{
Test-retest reliability and correlates of the Simple Physical Activity Questionnaire in Ugandan out-patients with psychosis
}

\author{
Davy Vancampfort ${ }^{1,2}$, David Basangwa ${ }^{3}$, Simon Rosenbaum ${ }^{4}$, Philip B Ward ${ }^{4,5}$, James Mugisha ${ }^{6,7}$
}

1. KU Leuven Department of Rehabilitation Sciences, Leuven, Belgium

2. University Psychiatric Centre KU Leuven, Kortenberg, Belgium

3. Butabika National Referral and Mental Health Hospital, Kampala, Uganda

4. School of Psychiatry, UNSW, Sydney, Australia;

5. Schizophrenia Research Institute, Ingham Institute of Applied Medical Research, Liverpool, Australia

6. Kyambogo University, Kampala, Uganda

\begin{abstract}
Background: In order to facilitate people with psychosis to increase their physical activity (PA) levels, a reliable measurement of these levels is of relevance.

Objectives: The primary aim of current study was to explore the test-retest of the Simple Physical Activity Questionnaire (SIMPAQ) in Ugandan outpatients with psychosis. A second aim was to explore correlates of the SIMPAQ, including demographic variables and antipsychotic medication dose.

Methods: Thirty-four women (33.9 \pm 8.0 years) and 21 men completed the SIMPAQ twice in a day. The test-retest reliability was assessed using Spearman Rho correlations coefficients. Differences in subgroups were analysed with Mann Whitney U tests.

Results: The SIMPAQ showed a good test-retest reliability with correlates ranging from $0.78(\mathrm{P}<0.001)$ for structured exercise to $0.96(\mathrm{P}<0.001)$ for walking. Women, non-smokers and those without HIV/AIDS showed higher incidental PA than men, smokers and those with HIV/AIDS.

Conclusion: The SIMPAQ is a reliable tool to assess PA and sedentary levels in Ugandan outpatients with psychosis. Men, smokers and those with HIV/AIDS appear to be at risk for lower incidental PA.

Keywords: Physical activity; sedentary lifestyle; psychosis.

DOI: https://dx.doi.org/10.4314/ahs.v20i3.49

Cite as: Vancampfort D, Basangwa D, Rosenbaum S, Ward PB, Mugisha J. Test-retest reliability and correlates of the Simple Physical Activity Questionnaire in Ugandan out-patients with psychosis Afri Health Sci. 2020;20(3): 1438-1445. https:/ / dx.doi. org/10.4314/abs.v20i3.49
\end{abstract}

\section{Introduction}

The benefits of physical activity are well established in people with psychotic disorders ${ }^{1-3}$. Physical activity and its structured subset, exercise, contribute to the prevention and treatment of cardiovascular diseases ${ }^{3,4}$, improve cognitive functioning and reduce mental health
Corresponding author:
Davy Vancampfort.
Tervuursevest 101
3001 Leuven,
Belgium
Tel.: +322 7580511
Fax: +32 27599879 .
Email: davy.vancampfort@kuleuven.be

symptom severity ${ }^{5,6}$. Despite these benefits, engaging people with psychosis in regular physical activity is a clinical challenge. A meta-analysis in people with schizophrenia demonstrated that the mean dropout rate from physical activity interventions is $26.7 \%{ }^{7}$. The most commonly reported barriers for initiating and maintaining physical activity are low mood and the presence of stress, followed by lack of support ${ }^{8}$. In order to facilitate people with psychosis to increase their physical activity levels, an accurate measurement of these levels at baseline and follow-up is of relevance ${ }^{9,10}$. Because detection of improvement without accurate measurement is impossible, ensuring that mental health clinicians have access to a brief, standardized clinical tool assessing key elements of physical activity participation is of high clinical significance ${ }^{11}$. 
In last years, there has been as well a rapid emergence of studies indicating that people with psychosis are more sedentary than the general population ${ }^{12}$. Also assesing sedentary levels are clinically relevant as higher levels of sedentariness in people with psychosis are associated with increased risk of metabolic syndrome ${ }^{13}$ and elevated c-reactive protein levels ${ }^{14}$.

Recently an international study in more than 1000 people with mental illness across 40 centers in 23 countries demonstrated that the Simple Physical Activity Questionnaire (SIMPAQ) ${ }^{11}$ is a reliable and valid instrument to assess physical activity and sedentary levels in this vulnerable population. The SIMPAQ is a 5 -item clinical tool developed to assess physical activity and sedentary behavior among populations at high risk for physical inactivity. It uses an interview format to estimate time spent in bed, time spent sedentary during waking hours, napping, time spent walking, in structured exercise participation, and in incidental or non-structured physical activity ${ }^{11}$. However, to date, its test-retest reliability has not been investigated yet in people with psychosis in low resource settings. Second, also correlates of physical activity and sedentary levels with psychotropic medication use (i.e. dose) in low resource settings is currently lacking. This is an important research gap since in most low resource settings mainly first generation antipsychotics are used ${ }^{15}$. Side effects of these first generation antipsychotics include motor disturbances and fatigue, which might influence physical activity participation and sedentary behavior ${ }^{16}$. Third, to date, there is no research on physical activity and sedentary behavior correlates in people with psychosis in sub-Saharan Africa (SSA). Physical activity levels might differ with levels in high-income countries due to the limited access to adquate physical activity facilities or to differences in daily life circumstances ${ }^{17}$. Considering the current gaps in the literature, the primary aim of current study is to explore the test-retest of the SIMPAQ in Ugandan outpatients with psychosis. A second aim was to explore correlates of the SIMPAQ, including demographic variables and antipsychotic medication dose.

\section{Methods \\ Participants and setting}

Over a 3-month period, outpatients with a DSM 5 diagnosis ${ }^{18}$ who had clinical diagnoses of psychosis including schizophrenia, schizoaffective disorder, bipolar disorder or depression with psychotic features and delusional disorders, as diagnosed by the treating psychia- trist of the Butabika National Referral Hospital, Kampala, Uganda, were invited to participate. Individuals were included if they were outpatients having at least a partial remittance in psychotic symptoms and were able to concentrate during the interview as determined by the treating psychiatrist. Since severe substance abuse and inpatient care might impair daily physical activity behavior, participants were excluded if they had a co-morbid DSM 5 diagnosis of substance abuse during the previous 6 months or were receiving inpatient care. Somatic exclusion criteria included self-reported evidence of significant cardiovascular, neuromuscular and endocrine disorders, which might prevent normal physical activity participation. A test-retest design was used to test the reproducibility of the SIMPAQ. Outpatients were asked to assess their physical activity and sedentary levels and again after a washout period of 6 hours on the same day. Re-tests were performed on the same day in order to avoid that patients had to return to the hospital. The study procedure was approved by the Scientific and Ethical Committee of Mengo Hospital, Kampala, Uganda and conducted in accordance with the principles of the Declaration of Helsinki. All participants gave their informed written consent. In case participants were not able to read or write, fingerprints were taken and a related witness signed the informed consent document.

\section{Sample size analysis}

An a-priori sample size calculation was conducted following recommendations for reliability analyses ${ }^{19}$. With a more than acceptable intraclass correlation coefficient (ICC) of 0.80 , and alpha of 0.05 and power of $0.8(\beta=0.2)$ it was established that 46 participants were required in the final analysis ${ }^{19}$. It was anticipated that approximately $10-20 \%$ of patients needed to be excluded, and $10-20 \%$ would refuse for motivational reasons. Therefore, a pre-specified sample size of 55 to 60 participants was utilized to account for these factors in order to ensure the final analysis was adequately powered.

\section{SIMPAQ}

The SIMPAQ ${ }^{11}$ is a 5 -item clinical tool to assess physical activity among populations at high risk for sedentary behavior. It uses an interview format to estimate time spent in bed (min/day), time spent sedentary during waking hours (min/day), time spent napping ( $\mathrm{min} /$ day), time spent walking ( $\mathrm{min} /$ day), time spent time spent in structured exercise ( $\mathrm{min} /$ day), and time spent in incidental or non-structured physical activity (min/ day) during the past week. 


\section{Anthropometric assessments}

Body weight and height were assessed in order to calculate the body mass index. Body weight was measured in light clothing to the nearest $0.1 \mathrm{~kg}$ using a SECA beam balance scale, and height to the nearest $0.1 \mathrm{~cm}$ using a wall-mounted stadiometer. Waist circumference (WC) was measured to nearest $1 \mathrm{~cm}$ at the level of the umbilicus and at the end of expiration with the participant upright and his/her hands by the side.

\section{Medication use}

We recorded the medication use. Anti-psychotic dose was recorded and converted into a daily chlorpromazine equivalent dosage according to an international consensus ${ }^{20}$. Mean dosages of specific mood stabilisers and antidepressants were reported when they were used by at least 10 participants.

\section{Smoking}

Participants were asked whether they smoked or not, and if so, how many cigarettes they smoke per day on average.

\section{Physical co-morbidity}

The presence of an ICD-10 ${ }^{21}$ physical co-morbidity was obtained from patients' medical records.

\section{Statistical analysis}

Continuous data were assessed for normality using the Shapiro-Wilk test and SIMPAQ data were found not to be normally distributed. Descriptive demographic data are therefore presented as mean and standard deviation and SIMPAQ data as median and interquartile range. The test-retest reliability was assessed using Spearman Rho correlations coefficients. Differences in SIMPAQ scores between men and women, between smokers and non-smokers, and between those with and without a chronic somatic condition were analysed with Mann Whitney $U$ tests and correlates with Spearman Rho correlations coefficients. A priori, a two sided level of significance was set at $\mathrm{P}<0.05$. Statistical analysis was performed using the statistical package SPSS version 23.0 (SPSS Inc., Chicago, IL).

\section{Results \\ Participants}

Out of 58 out-patients with psychosis, 55 met the inclusion criteria of which none declined to participate or dropped-out. Two patients had a co-morbid DSM 5 diagnosis of substance abuse which could affect physical activity levels while one patient was excluded due to medical reasons preventing normal physical activity participation in the previous week. The final sample consisted of 34 women (age $=33.9 \pm 8.0$ years; body mass index, $B M I=26.7 \pm 6.7 \mathrm{~kg} / \mathrm{m} 2$, waist circumference $=93.8 \pm 19.1)$ and 21 men (age $=37.6 \pm 12.2$ years; body mass index, $B M I=25.1 \pm 5.5 \mathrm{~kg} / \mathrm{m} 2$, waist circumference $=90.0 \pm 15.1)$. Seven participants $(13 \%)$ smoked a mean of $2.6 \pm 0.8$ cigarettes per day. Mean daily equivalent dosage of chlorpromazine $(n=51)$ was $279.6 \pm 169.8 \mathrm{mg} /$ day. Fourteen patients were treated with carbamazepine, four with lithium carbonate, five with valproic acid, three with fluoxetine and two with imipramine. Medical records indicated that $6(11 \%)$ patients were diagnosed with HIV/AIDS. No other physical co-morbidities were recorded. SIMPAQ data are presented in Table 1.

\section{Reliability of the SIMPAQ scores}

The SIMPAQ showed a good test - retest reliability with Spearman Rho correlates ranging from $0.78(\mathrm{P}<0.001)$ for structured exercise to $0.96(\mathrm{P}<0.001)$ for walking.

Table 1. Test-retest reliability of the SIMPAQ in Ugandan people with psychotic disorders $(\mathrm{n}=55)$

\begin{tabular}{lcccc}
\hline Variable & Assessment 1 & Assessment 2 & \multicolumn{2}{c}{ Spearman Rho } \\
\hline & & & R & P \\
\hline Bed (min/day) & $580(180)$ & $600(195)$ & 0.95 & $<0.001^{*}$ \\
Sedentary (min/day) & $660(157)$ & $645(180)$ & 0.91 & $<0.001^{*}$ \\
Napping (min/day) & $30(52)$ & $45(54)$ & 0.92 & $<0.001^{*}$ \\
Walking (min/day) & $18(23)$ & $15(24)$ & 0.96 & $<0.001^{*}$ \\
Exercise (min/day) & $0(0)$ & $0(0)$ & 0.78 & $<0.001^{*}$ \\
Incidental PA (min/day) & $60(97)$ & $60(120)$ & 0.94 & $<0.001^{*}$ \\
\hline
\end{tabular}

Data expressed as median (interquartile range), $\mathrm{PA}=$ physical activity, * significant when $\mathrm{P}<0.05$. 
Associations of the SIMPAQ scores

An overview of Spearman Rho correlations ( $r-$ and p-values) with age (years), BMI, waist (cm) and antip- sychotic medication use $(\mathrm{mg} /$ day) is presented in Table 2. Only more time spent in bed ( $\mathrm{min} /$ day) was significantly associated with a higher BMI and a higher waist circumference.

Table 2. Spearman Rho correlates of the SIMPAQ variables (retest) in Ugandan outpatients with psychosis $(\mathrm{n}=55)$

\begin{tabular}{|c|c|c|c|c|c|c|c|c|}
\hline \multirow[t]{2}{*}{ Variable } & \multicolumn{2}{|c|}{ Age (years) } & \multicolumn{2}{|c|}{ BMI } & \multicolumn{2}{|c|}{ Waist (cm) } & \multicolumn{2}{|c|}{ AP dose (mg/day) } \\
\hline & $\mathbf{r}$ & $\mathbf{P}$ & $\mathbf{r}$ & $\mathbf{P}$ & $\mathbf{R}$ & $\mathbf{P}$ & $\mathbf{r}$ & $\mathbf{P}$ \\
\hline Bed (min/day) & -0.16 & 0.23 & -0.32 & $0.02 *$ & -0.28 & $0.04 *$ & 0.16 & 0.26 \\
\hline Sedentary (min/day) & 0.13 & 0.36 & 0.18 & 0.18 & 0.25 & 0.08 & -0.18 & 0.22 \\
\hline Napping (min/day) & -0.10 & 0.47 & 0.006 & 0.97 & -0.19 & 0.16 & -0.05 & 0.74 \\
\hline Walking (min/day) & 0.02 & 0.86 & 0.002 & 0.99 & 0.003 & 0.98 & -0.16 & 0.27 \\
\hline Exercise (min/day) & 0.01 & 0.94 & 0.20 & 0.15 & 0.23 & 0.09 & -0.07 & 0.64 \\
\hline Incidental PA (min/day) & -0.13 & 0.34 & 0.04 & 0.80 & -0.02 & 0.89 & -0.02 & 0.88 \\
\hline
\end{tabular}

$\mathrm{BMI}=$ body mass index, $\mathrm{AP}=$ antipsychotic medication, $\mathrm{PA}=$ physical activity, ${ }^{*}$ significant when $\mathrm{P}<0.05$.

Differences in SIMPAQ scores between subgroups In Tables 3 to 5, differences in SIMPAQ scores were explored between men and women, smokers and non-smokers and those with and without HIV/AIDS.
Women (Table 3), non-smokers (Table 5) and those without HIV/AIDS (Table 5) showed higher incidental physical activity levels than men, smokers and those with HIV/AIDS respectively. Next to this, smokers spent less time napping than non-smokers (Table 4).

Table 3. Gender differences in SIMPAQ scores in Ugandan outpatients with psychosis

\begin{tabular}{lcccc}
\hline Variable & $\begin{array}{c}\text { Women } \\
(\mathbf{n}=\mathbf{3 4})\end{array}$ & $\begin{array}{c}\text { Men } \\
(\mathbf{n}=\mathbf{2 1})\end{array}$ & \multicolumn{2}{c}{ Mann Whitney U } \\
\hline & & & $\mathbf{U}$ & $\mathbf{P}$ \\
\hline Bed (min/day) & $540(225)$ & $600(195)$ & 300 & 0.48 \\
Sedentary (min/day) & $610(135)$ & $690311)$ & 280 & 0.28 \\
Napping (min/day) & $60(100)$ & $25(60)$ & 232 & 0.05 \\
Walking (min/day) & $15(22)$ & $17(40)$ & 333 & 0.91 \\
Exercise (min/day) & $0(0)$ & $0(6)$ & 292 & 0.22 \\
Incidental PA (min/day) & $90(101)$ & $5(60)$ & 182 & $0.004^{*}$ \\
\hline Data expressed as median (interquartile range), PA=physical activity, ${ }^{*}$ significant when $\mathrm{P}<0.05$.
\end{tabular}


Table 4. Differences in SIMPAQ scores between smokers and non-smokers

\begin{tabular}{lcccc}
\hline Variable & $\begin{array}{c}\text { Non-smokers } \\
(\mathbf{n}=\mathbf{4 8})\end{array}$ & $\begin{array}{c}\text { Smokers } \\
(\mathbf{n}=\mathbf{7})\end{array}$ & \multicolumn{2}{c}{ Mann Whitney U } \\
\hline & & & $\mathbf{U}$ & $\mathbf{P}$ \\
\hline Bed (min/day) & $600(180)$ & $570(285)$ & 141 & 0.95 \\
Sedentary (min/day) & $630(180)$ & $690(400)$ & 130 & 0.72 \\
Napping (min/day) & $45(96)$ & $0(37)$ & 62 & $0.02^{*}$ \\
Walking (min/day) & $15(24)$ & $10(25)$ & 97 & 0.21 \\
Exercise (min/day) & $0(0)$ & $0(2)$ & 134 & 0.80 \\
Incidental PA (min/day) & $60(120)$ & $0(60)$ & 71 & $0.04^{*}$ \\
\hline
\end{tabular}

Data expressed as median (interquartile range), $\mathrm{PA}=$ physical activity, ${ }^{*}$ significant when $\mathrm{P}<0.05$.

Table 5. Differences in SIMPAQ scores between Ugandan outpatients with psychosis with and without HIV/AIDS

\begin{tabular}{lcccc}
\hline Variable & $\begin{array}{c}\text { Without } \\
\text { HIV/AIDS } \\
(\mathbf{n = 4 9 )}\end{array}$ & $\begin{array}{c}\text { With } \\
\text { HIV/AIDS } \\
(\mathbf{n = 6})\end{array}$ & Mann Whitney U \\
& & & $\mathbf{U}$ & $\mathbf{P}$ \\
\hline Bed (min/day) & $600(180)$ & $660(300)$ & 109 & 0.71 \\
Sedentary (min/day) & $630(180)$ & $660(320)$ & 98 & 0.48 \\
Napping (min/day) & $45(92)$ & $60(45)$ & 122 & 0.99 \\
Walking (min/day) & $15(24)$ & $9(30)$ & 92 & 0.38 \\
Exercise (min/day) & $0(0)$ & $0(6)$ & 121 & 0.98 \\
Incidental PA (min/day) & $60(120)$ & $18(45)$ & 57 & $0.048^{*}$ \\
\hline Data expressed as median (interquartile range), PA=physical activity, *significant when $\mathrm{P}<0.05$
\end{tabular}

\section{Discussion}

To the best of our knowledge, the current study is the first to explore the test-retest reliability and correlates of the SIMPAQ in out-patients with psychosis in a low resource setting. The current data show that all variables showed excellent reliability with Spearman Rho correlates ranging from 0.78 for structured exercise to 0.96 for walking. For comparison purposes with other self-report physical activity assessments, reliability correlations in the physical activity literature range from 0.34 to $0.89^{22}$ while the only international study to date on the SIMPAQ including 1,010 participants with mental illness from 23 countries showed that test-retest correlations tanged from 0.63 for time spent in incidental activity $(\mathrm{p}<0.001)$ to 0.76 for time spent exercising and similarly for time spent walking (both $\mathrm{p}<0.001$ ), indicating acceptable to good reliability ${ }^{23}$. Given this comparability, researchers and clinical practitioners should consider the SIMPAQ suitable as a reliable surveillance tool to assess levels of physical activity and sedentary behavior among out-patients with psychosis in low-resourced settings. However, before the SIMPAQ can be recommended for standard use in low resource settings, research on its validity is needed. To this end, future research should compare the SIMPAQ scores with data obtained from criterion devices such as accelerometers. The Spearman rho correlation coefficient between the SIMPAQ moderate-vigorous physical activity and an objective assessment with an accelerometer was 0.25 for participants with available data $(n=617, \mathrm{p}<0.001)$ in the international SIMPAQ study ${ }^{23}$.

Our study is the first to explore correlates of the SIMPAQ in out-patients with psychosis and showed that age, gender nor the anti-psychotic medication dose were significantly associated with any SIMPAQ scores. In contrast, more time spent in bed (min/day) was significantly associated with a higher BMI and a higher 
waist circumference. Although due to the cross-sectional nature of this study causality cannot be determined, the data offer a platform for exploring associations between time spent in bed and cardio-metabolic risks in people with psychosis using longitudinal and interventional designs. Previous research in the general population did however not find support for the notion that long sleep ( $>8$ hours versus $7-8$ hours) is associated with metabolic risks ${ }^{24}$.

When looking at the physical activity levels, low median scores were reported for time spent exercising and time spent walking. A recent study evaluating mental health policies in sub-Saharan Africa ${ }^{25}$ demonstrated that although physical activity is becoming acknowledged as a major component in the management of severe mental illness, the potential is yet to be embraced. It is plausible that due to the strong focus on pharmacotherapy ${ }^{26}$ health care professionals are yet to be aware of the beneficial effects of aerobic exercise and walking for people with psychosis. Hence, the need to re-orient the current health care systems to embrace physical activity in the management of mental disorders is needed. A cheap, brief and reliable physical activity assessment such as administration of the SIMPAQ might facilitate such a culture change. The low walking and exercise levels and the limited variability might also explain why no significant correlates with demographic variables and anti-psychotic medication dose were found. In contrast, the median incidental physical activity levels were higher than the walking and exercise levels. A reason is that in the study setting (i.e. Butabika National Referral Hospital, Kampala, Uganda) out-patients are instructed to clean their rooms and wash their clothes themselves at home. Our data show that men, smokers and those with HIV/AIDS report significantly lower incidental physical activity levels. Therefore, in motivating out-patients with psychotic disorders to perform household chores in order to increase their physical activity levels special attention should be given to men, smokers and those with HIV. Recent research showed that in particular side effects of antiviral therapy (fatigue), HIV associated bodily pain, depression and opportunistic infections are important barriers for physical activity participation in those with $\mathrm{HIV}^{27}$.

Finally, our study showed that smokers spent significantly less time napping, a finding which is for example in contrast with a large-scale Japanese nationwide general population survey where smoking was associated with more frequent and long-duration napping ${ }^{28}$. More research is needed to explore associations between napping and other health behaviours in people with psychosis. In particular as a previous meta-analysis showed that a long daytime nap ( $\geq 60 \mathrm{~min} /$ day) is associated with a higher risk of cardiovascular disease (rate ratio $[R R]=1.82,95 \% \mathrm{CI}=1.22-2.71, \mathrm{P}=0.003)$ and all-cause mortality $(\mathrm{RR}=1.27,95 \% \mathrm{CI}=1.11-1.45, \mathrm{P}<0.001)$ compared with not napping ${ }^{29}$.

\section{Conclusion}

Our current study demonstrates the SIMPAQ is a reliable tool to assess physical activity and sedentary levels in Ugandan out-patients with psychosis. Before the tool can be recommended in routine clinical practice, a validity study to assess the levels against an objective criterion such as an accelerometer is needed.

\section{Acknowledgements}

None.

\section{Conflicts of interest}

The auhors declare that there is no conflict of interest to report.

\section{Funding}

This project was funded by Geestkracht VZW.

\section{Role of funding source}

The funding organization had no role in the research at any stage, nor influenced the decision to publish the article.

\section{References}

1. Rosenbaum S, Tiedemann A, Sherrington C, Curtis J, Ward PB. Physical activity interventions for people with mental illness: a systematic review and meta-analysis. Journal of Clinical Psychiatry. 2014;75(9):964-74. doi: 10.4088/JCP.13r08765.

2. Firth J, Cotter J, Elliott R, French P, Yung A. A systematic review and meta-analysis of exercise interventions in schizophrenia patients. Psychological Medicine. 2015;45(7):1343-61. doi: 10.1017/S0033291714003110. 3. Vancampfort D, Rosenbaum S, Ward P, Stubbs B. Exercise improves cardiorespiratory fitness in people with schizophrenia: A systematic review and meta-analysis. Schizophrenia Research. 2015;169(1-3):453-7. doi: 10.1016/j.schres.2015.09.029.

4. Stubbs B, Rosenbaum S, Vancampfort D, Ward PB, Schuch FB. Exercise improves cardiorespiratory fit- 
ness in people with depression: A meta-analysis of randomized control trials. Journal of Affective Disorders. 2016;190:249-53. oi: 10.1016/j.jad.2015.10.010.

5. Rosenbaum S, Tiedemann A, Ward PB, Curtis J, Sherrington $\mathrm{C}$. Physical activity interventions: an essential component in recovery from mental illness. British Journal of Sports Medicine. 2015; 49(24):1544-5. doi: 10.1136/ bjsports-2014-094314.

6. Firth J, Stubbs B, Rosenbaum S, Vancampfort D, Malchow B, Schuch F, et al. Aerobic exercise improves cognitive functioning in people with schizophrenia: a systematic review and meta-analysis. Schizophrenia Bulletin. 2017;43(3):546-56. doi: 10.1093/schbul/sbw115.

7. Vancampfort D, Rosenbaum S, Schuch FB, Ward PB, Probst M, Stubbs B. Prevalence and predictors of treatment dropout from physical activity interventions in schizophrenia: a meta-analysis. General Hospital Psychiatry. 2016;39:15-23 PubMed . doi: 10.1016/j.genhosppsych.2015.11.008.

8. Firth J, Rosenbaum S, Stubbs B, Gorczynski P, Yung A, Vancampfort D. Motivating factors and barriers towards exercise in severe mental illness: a systematic review and meta-analysis. Psychological Medicine. 2016:1-13. doi:10.1017/S0033291716001732

9. Vancampfort D, Stubbs B, Ward P, Teasdale S, Rosenbaum $\mathrm{S}$. Integrating physical activity as medicine in the care of people with severe mental illness. Australian and New Zealand Journal of Psycbiatry. 2015;49(8):681-2. doi: 10.1177/0004867415590831.

10. Vancampfort D, Stubbs B, Ward PB, Teasdale $\mathrm{S}$, Rosenbaum S. Why moving more should be promoted for severe mental illness. The Lancet Psychiatry. 2015;2(4):295. doi: 10.1016/S2215-0366(15)00099-1.

11. Rosenbaum S, Ward PB. The Simple Physical Activity Questionnaire. The Lancet Psychiatry. 2016;3(1):e1. doi: 10.1016/S2215-0366(15)00496-4.

12. Stubbs B, Williams J, Gaughran F, Craig T. How sedentary are people with psychosis? A systematic review and meta-analysis. Schizophrenia Research. 2016;176(23):431-440. doi: 10.1016/j.schres.2016.05.017.

13. Vancampfort D, Probst M, Knapen J, Carraro A, De Hert M. Associations between sedentary behaviour and metabolic parameters in patients with schizophrenia. Psychiatry Research. 2012;200(2):73-8. doi: 10.1016/j. psychres.2012.03.046

14. Stubbs B, Gardner-Sood P, Smith S, Ismail K, Greenwood K, Farmer R, et al. Sedentary behaviour is associated with elevated C-reactive protein levels in people with psychosis. Schizophrenia Research. 2015;168(1):4614. doi: 10.1016/j.schres.2015.07.003

15. Mugisha J, Ssebunnya J, Kigozi FN. Towards un- derstanding governance issues in integration of mental health into primary health care in Uganda. International Journal of m-Mental Health Systems. 2016;10(1):1. doi: 10.1186/s13033-016-0057-7.

16. Vancampfort D, Knapen J, Probst M, van Winkel R, Deckx S, Maurissen K, et al. Considering a frame of reference for physical activity research related to the cardiometabolic risk profile in schizophrenia. Psychiatry Research. 2010;177(3):271. doi: 10.1016/j.psychres.2010.03.011.

17. Stubbs B, Koyanagi A, Schuch F, Firth J, Rosenbaum S, Gaughran F, et al. Physical activity levels and psychosis: a mediation analysis of factors influencing physical activity target achievement among 204186 people across 46 low-and middle-income countries. Schizophrenia Bulletin. 2017; 3(3):536-545. doi: 10.1093/ schbul/sbw111..

18. American Psychiatric Association. Diagnostic and Statistical Manual of Mental Disorders (DSM-5). American Psychiatric Association, Washington, DC2013.

19. Walter S, Eliasziw M, Donner A. Sample size and optimal designs for reliability studies. Statistics in Medicine. 1998;17(1):101-10. doi: 10.1002/(SICI)10970258(19980115) 17:1<101::AID-SIM727>3.0.CO;2-E

20. Gardner DM, Murphy AL, O'Donnell H, Centorrino F, Baldessarini RJ. International consensus study of antipsychotic dosing. American Journal of Psychiatry. 2010;167:686-93. doi: 10.1176/appi.ajp.2009.09060802. 21. World Health Organization. The ICD-10 Classification of Mental and BehaviouralDisorders- Diagnostic Criteria for Research. Geneva: World Health Organization; 1993.

22. Sallis JF, Saelens BE. Assessment of physical activity by self-report: status, limitations, and future directions. Research Quarterly for Exercise and Sport. 2000;71(sup2):114.

23. Rosenbaum A, Morell R, Abdel-Baki A, Achmadpana M, Baie L, Bauman A, et al. Assessing physical activity in people with mental illness: 23-country reliability and validity of the Simple Physical Activity Questionnaire (SIMPAQ). BMC Psychiatry 2020; doi: 10.21203/ rs. $2.12937 / \mathrm{v} 2$

24. Iftikhar IH, Donley MA, Mindel J, Pleister A, Soriano S, Magalang UJ. Sleep Duration and Metabolic Syndrome: An Updated Dose-Risk Meta-analysis. Annals of the American Thoracic Society. 2015(ja).

25. Vancampfort D, Stubbs B, De Hert M, du Plessis C, Gbiri CAO, Kibet J, et al. A systematic review of physical activity policy recommendations and interventions for people with mental health problems in Sub-Saharan African countries. Pan African Medical Journal. 
2017;26(104). doi: 10.11604/pamj.2017.26.104.10051. 26. Mugisha J, Hert MD, Stubbs B, Basangwa D, Vancampfort D. Physical health policies and metabolic screening in mental health care systems of Sub-Saharan African countries: a systematic review. International Journal of Mental Health Systems. 2017; 11:31. doi: 10.1186/ s13033-017-0141-7.

27. Vancampfort D, Mugisha J, Richards J, Hert MD, Probst M, Stubbs B. Physical activity correlates in people living with HIV/AIDS: a systematic review of 45 studies. Disability \& Rehabilitation. 2018; 40(14):16181629.
28. Furihata R, Kaneita Y, Jike M, Ohida T, Uchiyama M. Napping and associated factors: a Japanese nationwide general population survey. Sleep Medicine. 2016;20:72-9 PubMed . doi: 10.1016/j.sleep.2015.12.006.

29. Yamada T, Hara K, Shojima N, Yamauchi T, Kadowaki T. Daytime napping and the risk of cardiovascular disease and all-cause mortality: a prospective study and dose-response meta-analysis. American Academy of Sleep Medicine; 2015; 38(12): 1945-1953. doi: 10.5665/ sleep. 5246. 